\section{New Alzheimer's disease screening test performs well in UK outpatient settings}

\section{QUESTION}

Question: How effective is the TYM ('test your memory') cognitive test in detecting Alzheimer's disease?

Patients: 139 people attending a memory clinic: 31 people with non-Alzheimer's degenerative dementias, 108 people with Alzheimer's disease or amnestic mild cognitive impairment (85 with National Institute of Neurological and Communicative Disorders and Stroke-Alzheimer's Disease and Related Disorders Association criteria diagnosis and 23 with amnestic mild cognitive impairment). 94 people (mean age 69 years) were included in the analysis for Alzheimer's disease as nine people with amnestic mild cognitive impairment scored poorly on the Addenbrooke's Cognitive Examinationrevised (ACE-R) and were regarded as likely to progress to Alzheimer's disease. The 14 remaining people with amnestic mild cognitive impairment were analysed separately. People with cognitive problems caused by depression were excluded. 540 control participants aged 18-95 years were recruited from relatives taking people to the memory clinic, medical outpatients from two other hospitals and some dermatology outpatients. People with memory problems, a history of neurological disease or brain injury were excluded.

Setting: Three hospital outpatient departments, Cambridge; March-December 2007.

Test: The TYM (http://tymtest.com/) comprises 11 tasks: orientation (10 points), semantic knowledge (3 points), verbal fluency (4 points), naming (5 points), ability to copy a sentence ( 2 points), calculation ( 4 points), similarities (4 points), visuospatial abilities (two tasks, total 7 points) and recall of a copied sentence ( 6 points). Ability to do the test is the final task and scored 5 points. Total score (maximum 50 ) is calculated by adding the scores together. The TYM is presented on double sided card, with spaces for users to complete the test.

Diagnostic standard: ACE-R, that includes the Mini-Mental State Examination (MMSE)

Outcomes: Sensitivity, specificity, positive predictive value and negative predictive value.

\section{METHODS}

Design: Diagnostic cohort study.

\section{MAIN RESULTS}

Mean TYM score of control participants was 47/50. Mean TYM score of participants with Alzheimer's disease was 33/50. Mean TYM score of participants with non-Alzheimer's dementias was 39/50. There were significant correlations between all scores in patients with Alzheimer's disease (TYM vs MMSE, $\mathrm{R}^{2}=0.51$, $\mathrm{p}<0.01$; TYM vs ACE- $R, \mathrm{R}^{2}=0.66, \mathrm{p}<0.01$; ACE-R vs MMSE, $\left.\mathrm{R}^{2}=0.770, \mathrm{p}<0.001\right)$. Three age matched controls $(\mathrm{n}=282)$ were randomly selected for each patient with Alzheimer's disease and used to calculate a receiver operating curve (see table). A TYM score cut-off of $\leq 42$ predicts mild Alzheimer's disease with a sensitivity of $93 \%$ and specificity of $86 \%$. Assuming a $10 \%$ prevalence of Alzheimer's disease and a cut-off $\leq 42$, the positive predictive value of the TYM was $42 \%$ and the negative predictive value $99 \%$. The TYM had greater sensitivity for detecting Alzheimer's disease compared with the MMSE ( $93 \%$ vs $52 \%$ ).

\begin{tabular}{|c|c|c|c|c|}
\hline Score & Sensitivity & False negative rate & Specificity & False positive rate \\
\hline$\leq 45$ & 97 & 3 & 75 & 25 \\
\hline$\leq 44$ & 96 & 5 & 81 & 19 \\
\hline$\leq 43$ & 94 & 6 & 83 & 17 \\
\hline$\leq 42$ & 93 & 7 & 86 & 14 \\
\hline$\leq 41$ & 87 & 13 & 91 & 9 \\
\hline$\leq 40$ & 83 & 17 & 92 & 8 \\
\hline$\leq 39$ & 81 & 19 & 95 & 5 \\
\hline$\leq 38$ & 75 & 25 & 96 & 4 \\
\hline
\end{tabular}

\section{CONCLUSIONS}

TYM is a valid new screening test for detecting Alzheimer's disease.

\section{ABSTRACTED FROM}

Brown J, Pengas G, Dawson K, et al. Self administered cognitive screening test (TYM) for detection of Alzheimer's disease: cross sectional study. BMJ 2009;338:b2030.

Correspondence to: J Brown, Department of Neurology, Addenbrooke's Hospital, Cambridge CB2 200, UK; jmb75@medschl.cam.ac.uk

Source of funding: Alzheimer's Research Trust (UK), Cambridge Commonwealth Trust, Stroke Association.
It must be unusual for a paper describing the development of a questionnaire to be published in a general journal. How did this study, careful and detailed as it is, make it into the BMJ? We suspect it is because the $B M J$ editors have an eye for any clinical research that is potentially very important.

The TYM ('test your memory') cognitive test, described by the authors as a 'powerful and valid screening test for the detection of Alzheimer's disease', could become a useful tool for non-specialists when assessing patients who complain of problems with their memory. This can be a difficult and uncomfortable task as it is sometimes the first step towards diagnosis of an untreatable neurodegenerative disease.

TYM appears to have robust psychometric properties with a sensitivity of $93 \%$ and specificity of $86 \%$ in detecting mild Alzheimer's disease in the subjects in this study. This is a substantial improvement on the widely used Mini-Mental State Examination (MMSE). Unlike the MMSE, it can be completed quickly and efficiently by patients with only minimal supervision. It also has a wider scoring range than the MMSE and so may be more helpful in the monitoring of diagnosed patients' improvement or decline.

However, as was pointed out in the correspondence that followed this paper, there would be an unacceptably high number of false positive tests if this instrument were to be used uncritically by clinicians or, even more so, if it was downloaded from the internet and used as a screening test by patients and families. Because of this possibility, the TYM is available only to registered health practitioners from Brown and colleagues' useful website.
It remains to be seen where this measure will find its niche as a clinical tool. The Department of Health has a strategy to encourage the early diagnosis of dementia with a view to improving the quality of ongoing care. The TYM may assist patients' care pathway so long as it is used with caution as one component of the initial clinical investigation and subsequent monitoring. We would like to see formal testing in a wider range of illnesses, and we need more clinical experience with this new instrument, but in our own ward and clinics, it looks like the days of the MMSE are numbered.

Sameer Jauhar, MRCPsych

Anthony Pelosi, FRCPsych

Hairmyres Hospital, East Kilbride, UK

Competing interests: None 